\title{
Perspectiva de los centros de escritura en Colombia*
}

\author{
Gerzon Yair Calle Álvarez**
}

Recibido: 12 de julio de 2016

Evaluado: 15 de septiembre de 2016

Aceptado: 8 de octubre de 2016

\section{Resumen}

En este artículo se describen 19 centros de escritura (CE) de Colombia, a partir de la información que aparece en sus páginas web y de publicaciones que se han generado sobre las experiencias de estos espacios dentro de las instituciones en que se instauran. La metodología utilizada fue la investigación documental. Para comenzar, se presenta una introducción sobre la lectura y escritura en Colombia; luego, el texto se centra en la descripción de los centros de escritura en la educación superior en el contexto de Colombia, sin embargo se aborda un CE dentro de la educación básica y media, que se ha identificado. Al final, se plantea la perspectiva general de los centros de escritura en el contexto colombiano. Como conclusión, se afirma que los centros de escritura en Colombia son una estrategia reciente, que se ha venido pensando con el propósito de fortalecer los procesos de escritura académica en los estudiantes.

Palabras clave: centro de escritura, escritura académica, lectura académica. 


\title{
Perspective of Writing Centers in Colombia
}

\begin{abstract}
This article describes 19 writing centers in Colombia based on the information that appears on their web pages and publications that have been generated on the experiences of these spaces within the institutions where they are established. The methodology used was documentary research. To begin, an introduction on reading and writing is presented in Colombia; Then focuses on the description of writing centers in the university in the context of Colombia, however, it addresses a writing center in primary and secondary education, which was identified. In the end, the general perspective of the Writing Centers is presented in the Colombian context. In conclusion, it is stated that writing centers in Colombia are a recent strategy that has been considered in order to strengthen the academic writing processes in students.
\end{abstract}

Received: July 12, 2016

Evaluated: September 15, 2016

Keywords: Writing center, academic writing, academic reading. 


\section{Perspectiva dos centros de escrita na Colômbia}

Recebido: 12 de julho de 2016

Avaliada: 15 de setembro de 2016

Aceito: 8 de outubro de 2016

\section{Resumo}

Neste artigo descrevem-se 19 centros de escrita (CE) da Colômbia, a partir da informação que aparece nas suas paginas da internet e das publicações que tem se gerado sobre as experiências destes espaços dentro das instituições nas quais se instauram. A metodologia utilizada foi a pesquisa documental. No começo, se apresenta uma introdução sobre a leitura e a escritura na Colômbia; depois, o texto foca-se na descrição dos centros de escrita na educação superior no contexto da Colômbia, porem, aborda-se um CE na educação básica e média que se tem identificado. Ao final, se expõe a perspectiva geral dos centros de escrita no contexto colombiano. Como conclusão, afirma-se que os centros de escrita na Colômbia são uma estratégia recente, que se tem pensado com o objetivo de fortalecer os processos de escrita acadêmica nos Estudantes.

Palavras-chave: Centro de escrita, escrita acadêmica, leitura acadêmica. 


\section{INTRODUCCIÓN}

En Colombia, el fortalecimiento de los procesos de lectura y escritura, en los diferentes ámbitos educativos, ha sido un interés de los entes gubernamentales y la comunidad académica. Se ha reconocido que el dominio de las habilidades comunicativas supera los procesos de codificación y decodificación de la lengua, e implica procesos de incorporación y participación de las personas en la sociedad. Así, las instituciones educativas, ya sean de educación básica, media o superior, tienen una responsabilidad en desarrollar estrategias para el fortalecimiento de las prácticas de lectura y escritura en los integrantes de sus comunidades.

En el contexto colombiano, desde el Ministerio de Educación Nacional se creó el Plan Nacional de Lectura y Escritura (PNLE), que tiene entre sus objetivos consolidar la escuela y la biblioteca escolar como espacios propicios para la formación de lectores y escritores, con apoyo de las familias. Se busca que los beneficiarios directos sean los estudiantes que se encuentran en la educación básica; sin embargo, se requiere el apoyo de los directivos, profesores, padres de familia y partes interesadas, de las instituciones educativas.

En el PNLE se ha considerado la incorporación de recursos análogos y digitales. Así, dentro de la línea de materiales, se ha venido dotando a las instituciones educativas de libros de papel y de recursos digitales para la lectura y la escritura. Los materiales llegan a las bibliotecas escolares para acceso y uso de los integrantes de las comunidades educativas; se busca que estos espacios no sean solo un lugar de almacenamiento de materiales, sino lugares vivos para la promoción de la lectura y escritura.

En la misma línea, las instituciones educativas han desarrollado estrategias, como programas de lectura y escritura, actualización de programas y planes de áreas, talleres, concursos de escritura, maratones de lectura. Estos recursos pueden responder a realidades institucionales, a interés de la comunidad educativa, a iniciativas de profesores, a proyectos gubernamentales, pero, en general, estarán enfocadas en fortalecer las prácticas de lectura y escritura en los estudiantes.

Toloza, Barletta y Moreno (2013) realizaron una investigación sobre la enseñanza y aprendizaje de la lectura y la escritura en una institución educativa de la región Caribe colombiana. En la segunda fase del estudio diseñaron una estrategia de acompañamiento para las clases de Lengua Castellana, mediante la teoría socioconstructivista, basada en la pedagogía de géneros. Para su desarrollo, asumieron el cuento como el soporte para el avance de la propuesta de intervención. Además, durante el estudio conformaron una comunidad de práctica que estuvo compuesta por seis profesores universitarios, la profesora del área de Lengua Castellana y el jefe de área de la institución educativa.

Dentro de las conclusiones de la intervención, se planteó que la enseñanza basada en géneros es una buena opción para mejorar los procesos de lectura y escritura en instituciones en que los estudiantes presenten deficiencia en estos aspectos; sin embargo, es muy importante el acompañamiento a los profesores en sus prácticas de aula, con el fin de que se actualicen dichas prácticas. 
En la educación superior, también se han visibilizado estrategias para el fortalecimiento de las prácticas de lectura y escritura. González y Vega (2013) realizaron un estudio sobre prácticas de lectura y escritura presentes en cinco cursos de diferentes programas de la Universidad Sergio Arboleda (sede Bogotá). En los resultados se evidenció la preocupación de la comunidad educativa por el tema de la lectura y la escritura dentro de la Universidad, pero se reconocieron pocas acciones que le aportaran al campo. En las conclusiones se plantea la importancia de pensar y diseñar estrategias para el fortalecimiento de la lectura y la escritura que correspondan a las disciplinas; también, se afirma que la colaboración y construcción de estrategias entre los profesores es una oportunidad para la creación de una comunidad de enseñanza, en la que se pueda aprovechar el potencial de la lectura y la escritura para el desarrollo del pensamiento crítico en los estudiantes.

En la misma dirección, algunas instituciones de educación superior han incorporado dentro de sus dinámicas los centros de escritura (CE). Los CE, en el contexto colombiano, son una estrategia que no lleva más de diez años en las instituciones de educación superior. El primer CE que se fundó en Colombia fue el de la Pontificia Universidad Javeriana, en Cali, en el 2008. Los referentes que se han asumido para el diseño, montaje y evaluación de los CE han sido tomados del modelo anglosajón.

Núñez (2013) realizó un rastreo a nueve CE de Iberoamérica, desde la información de cada una de sus páginas web: tres de Argentina, dos de España, dos de Colombia, uno de México y uno de Perú. Dentro de los elementos orientados de los centros de escritura, encontró los siguientes elementos: 1. modalidades: presencial o virtual; 2. destinatarios: profesores y estudiantes; 3. objetivo: desarrollar la competencia comunicativa en la universidad; 4 . propósitos: formación, información, asesoramiento, investigación, promoción; 5. talleres de escritura académica; 6 . asesorías presenciales y virtuales; 7 . centros de recursos.

Los dos centros de escritura de Colombia que retoma Núñez (2013) fueron: el Centro de Español (http://programadeescritura.uniandes.edu.co/), de la Universidad de los Andes (Bogotá); y el Centro de Escritura Javeriano (http://centrodeescritura.javerianacali.edu.co/), de la Pontificia Universidad Javeriana de Cali (Cali). Algunas de las conclusiones del rastreo fueron las siguientes: 1 . aunque los centros de escritura nacieron en el contexto anglosajón, Iberoamérica ha realizado un avance en esta materia, al responder a las exigencias de alfabetización académica de esta región; 2. comenzar a tener centros de escritura en Iberoamérica podrá ser un primer paso para crear redes en la región para el mejoramiento de la escritura académica en la educación superior.

\section{REFERENTES METODOLÓGICOS}

Para la construcción del panorama de los CE en Colombia, se aplicó la investigación documental. Según Alfonzo (1994), la investigación documental es un proceso sistemático de indagación, recolección, organización, análisis e interpretación de información en torno a un tema específico. Esta tiene la particularidad de utilizar como fuente primaria documentos escritos en diferentes formas: 
análogos y digitales. En este estudio se usaron ambos tipos de textos: análogos, desde los libros de consulta y artículos de revistas; digitales, acceso a páginas web de los CE.

Para la presente investigación se determinó seleccionar un corpus de 18 CE que operan en espacios de la educación superior, y un CE de la educación media, en el territorio colombiano. Vale aclarar que la tradición de estos espacios se ha dado en la universidad, y ha sido poco explorado en la educación inicial y media. Las fuentes documentales que se utilizaron fueron las páginas web de cada uno de los CE y las publicaciones sobre estos centros en el contexto colombiano. Se identificó que todos los que hacen parte del corpus tienen un espacio en la web. En la búsqueda en las bases de datos se identificaron 19 fuentes análogas, que presentan resultados de investigaciones desarrolladas sobre servicios y acciones de los CE en los contextos en que se instauran.

Con el propósito de desarrollar un proceso coherente y sistemático, para buscar un acercamiento profundo a la información recolectada, se plantearon las siguientes acciones: 1. identificación de las fuentes de información; para ello se creó un listado con las páginas web de los CE y la bibliografía encontrada, en un archivo de Microsoft Excel. 2. Lectura interpretativa y consolidación de información recolectada; se creó una ficha bibliográfica y de práctica, en la que se identificó la referencia, la dirección web del CE y un resumen de los componentes del CED. 3. Redacción del perfil de cada CE; se tuvo presente la mirada que estos espacios tienen sobre la lectura y la escritura, los servicios ofrecidos y las interacciones que se establecen con los integrantes de las comunidades educativas. 4. Línea de base; perspectiva de los CE en el contexto colombiano, desde la ubicación geográfica, estructura organizacional, concepciones de la escritura, propósitos misionales, población beneficiada, servicios, uso de TIC.

\section{Centro de Español, de la Universidad de los Andes, Bogotá}

\section{http://programadeescritura.uniandes.edu.co/}

El Centro de Español hace parte de la Vicerrectoría de Asuntos Académicos, de la Universidad de los Andes. Es una estrategia pensada para el desarrollo de las competencias analíticas, reflexivas, argumentativas y de pensamiento crítico de los estudiantes, mediante la escritura (Escallón Largacha, 2015). Se concibe la escritura como una competencia en la formación de estudiantes autónomos y ciudadanos comprometidos. Dentro del Centro de Español se cuenta con un plan denominado 'Programa de escritura', que tiene tres líneas de trabajo: cursos E, curso de español y Centro de Escritura. Sus destinatarios son los profesores y estudiantes, y para esta población existen unas estrategias y recursos determinados. Los profesores cuentan con recursos en la página web, sobre todo para aquellos que imparten una asignatura de escritura intensiva, plantillas de evaluación o matrices de textos argumentativos y textos expositivos (reseñas y resúmenes).

Los cursos E son asignaturas de las distintas facultades de la Universidad, que tienen un programa atravesado por la escritura académica. Están pensados para que los estudiantes que elijan estos cursos 
desarrollen y demuestren comprensión propia de los textos de la disciplina (Montealegre \& Morales, 2015). Para que una asignatura adquiera el título de curso E, el programa deberá estar pensado desde las relaciones que se establecen con la escritura, y debe ser revisado y avalado por el Centro de Español.

El curso de español tiene como propósito desarrollar en los estudiantes las competencias de lectura y escritura básicas para afrontar los retos de la educación superior. Es un curso obligatorio para todos los estudiantes de pregrado de la Universidad de los Andes. Los profesores del curso, en su mayoría, son estudiantes de maestría o doctorado de Literatura o Ciencias Sociales, y reciben una formación específica antes de comenzar a desempeñarse como profesores del curso. El enfoque del curso es comunicativo, parte de la lectura de tres textos argumentativos y académicos, que servirán de modelo para la escritura de cinco escritos por parte de los estudiantes (Forero Gómez, 2015).

Por otra parte, los estudiantes y profesores podrán asistir al $\mathrm{CE}$, donde encontrarán un tutor que los orientará en el proceso de construcción de sus textos. Como afirma Lara Negrette (2015), la tutoría es un espacio de aprendizaje privilegiado para desarrollar competencias argumentativas y analíticas, por medio de la escritura. La cita para la tutoría se solicita por medio de la página web del Centro de Español y tiene una duración de 50 minutos, máximo. Al finalizar, el tutor diligencia un formulario virtual para resumir la tutoría, y el estudiante o profesor que la solicitó, evalúa el servicio. Los tutores son estudiantes de posgrado, quienes después de pasar una prueba escrita, reciben una formación para desempeñarse como tutores.

Finalmente, los estudiantes cuentan con recursos en la página web, relacionados con información y ejercicios sobre cómo escribir una tesis, conclusiones de un texto argumentativo, nociones sobre el párrafo, cómo manejar la ansiedad al escribir, cómo hacer una guía de lectura, preguntas para organizar la lectura de un texto, entre otros. Además, el Centro de Español ejecuta estrategias sobre asuntos puntuales; por ejemplo, el campamento de tesis, durante el semestre 2016-2, que buscaba acompañar a los estudiantes de posgrado durante 40 horas en la escritura de sus tesis.

\section{Centro de Escritura Javeriano, de la Pontificia UNIVERSIDAD JAVERIANA, SEDE Cali}

http://centrodeescritura.javerianacali.edu.co/

El Centro de Escritura Javeriano fue creado en el 2008 (Molina Natera, 2015); depende del Departamento de Comunicación y Lenguaje de la Facultad de Humanidades y Ciencias Sociales de la Pontificia Universidad Javeriana de Cali. Dentro de sus objetivos, contempla ser un espacio de aprendizaje inclusivo y colaborativo, desarrollar destrezas de escritura en la comunidad educativa, constituirse en un programa de generación de conocimiento y ayudar a los estudiantes a desarrollarse como pensadores críticos, por medio de la escritura de sus propios textos.

Los tutores son estudiantes de pregrado de la Universidad. Sin embargo, para ser tutor 
del CE, estos deben contar con una recomendación escrita de uno de los profesores de la Universidad, en la que se certifiquen las capacidades escriturales y de trabajo en equipo con las que cuenta el estudiante. Luego, los estudiantes deben realizar un curso de un semestre, y al final de este se determina quiénes pasarán a ser parte del grupo de tutores.

Los destinatarios del centro son los profesores y los estudiantes. Los profesores pueden acceder a asistencias para diseñar e implementar estrategias de aula enfocadas a la escritura; así, uno de los tutores puede acompañar al profesor a sus clases, y conformar equipos de pares académicos. Los estudiantes cuentan con tutorías y talleres de escritura académica; además, por medio de la página web, pueden acceder a recursos que les ayudan a aclarar sus dudas sobre determinados asuntos durante la escritura. Vale anotar que desde el CE se fomenta un taller de lectura en la Universidad.

Molina Natera (2015) realizó un estudio de caso en el marco de la investigación "¿Para qué se lee y se escribe en las universidades de Colombia? Un aporte a la consolidación de la cultura académica del país". En la investigación se seleccionaron prácticas de lectura y escritura destacadas dentro 17 universidades. El único CE que se presentó fue el Centro de Escritura Javeriano. Dentro de las conclusiones del estudio, se encontró que los textos revisados por el CE presentan una mejoría en el producto final y permite a los profesores planear las tareas de escritura como un proceso; el CE, además, ha logrado crear conciencia en la comunidad educativa sobre la importancia de la escritura.

\section{Centro de Escritura Javeriano, de la Pontificia UNIVERSIDAD JAVERIANA, SEDE Bogotá}

https://cejaveriana.wordpress.com/

El Centro de Escritura Javeriano hace parte del Departamento de Lenguas de la Facultad de Comunicación y Lenguaje de la Pontificia Universidad Javeriana, sede Bogotá; sin embargo, hay un trabajo interdisciplinar y de acompañamiento con las facultades de Educación y Psicología. Inició sus labores en el 2011. En el CE se brinda acompañamiento a estudiantes de pregrado y posgrado, y a profesores, en la lectura, y se dan sugerencias, por parte de un par académico, de los textos que estos producen.

Los tutores son estudiantes de pregrado o posgrado de cualquiera de los programas que ofrece la Pontificia Universidad Javeriana, que han cursado una asignatura llamada Tutores, Centro de Escritura Académica, durante un semestre. En este tiempo se les brindan herramientas a los futuros tutores para que puedan desempeñar las funciones en el acompañamiento y fortalecimiento de las producciones académicas de otros estudiantes. En tutoría se puede desarrollar un acompañamiento sobre los siguientes aspectos: documentación, planeación de un texto, estrategias para consultar y organizar la información, referenciación y citación, entre otros.

Moreno y Baracaldo (2015) realizaron un estudio sobre el acompañamiento que realizó el Centro de Escritura Javeriano, sede Bogotá, a las prácticas de lectura y escritura de los estudiantes del primer semestre del programa de Contaduría, dentro de la asignatura 
Teoría del Aseguramiento. En las conclusiones se presenta que se ha buscado que los profesores de la asignatura contextualicen las lecturas y que reconozcan la escritura como una posibilidad para el aprendizaje, y no solo como un instrumento evaluativo; además, se orientó a los profesores para que tomaran decisiones didácticas sobre la lectura y la escritura de los textos de la disciplina que orientan.

González (2015) afirma que los principales logros del Centro de Escritura Javeriano, sede Bogotá, son: 1. diálogo interdisciplinario entre los profesores de las facultades de Educación, Psicología y Comunicación; 2. formación de 28 estudiantes como pares académicos; 3 . avances con otras facultades para la incorporación de los procesos de lectura y escritura dentro de diferentes materias; 4 . apoyo a la escritura de tesis de los estudiantes de posgrado; 5 . intercambio de experiencias con universidades nacionales e internacionales; 6. sistematización de la experiencia de la formación de pares académicos.

En Centro de Escritura Javeriano, de Bogotá, cuenta con una red social en Twitter (@CEJaverianaB), la cual se mantuvo muy activa durante sus inicios; sin embargo, a partir de mediados del 2015, no se volvió a registrar actividad. Por otra parte, en su página web hay información sobre qué es el $\mathrm{CE}$, sus integrantes y algunos escritos realizados por los tutores; la única forma de interacción posible sería por medio de comentarios sobre estos aspectos, pero a la fecha no se evidencia ningún comentario por parte de la comunidad universitaria.

\section{Centro de Escritura \\ UNIMINUTO, DE LA \\ CORPORACIÓN UNIVERSITARIA Minuto de Dios, sede Bogotá}

\author{
http://www.uniminuto.edu/web/fcc/centro \\ -de-escritura
}

El Centro de Escritura Uniminuto hace parte de la Facultad de Ciencias de la Comunicación, de la Corporación Universitaria Minuto de Dios, sede Bogotá. Se inició su funcionamiento en el 2011. Este Centro se presenta como una estrategia pedagógica para fortalecer los procesos de lectura y escritura de los estudiantes, y busca que las prácticas de escritura vayan más allá de la valoración de una nota en determinada asignatura. Los estudiantes cuentan con dos recursos: asesorías personalizadas y talleres (de ortografía o redacción).

Uno de los pilares del Centro de Escritura Uniminuto es el trabajo colaborativo, reflejado en la tutoría personalizada entre pares académicos. Los tutores son estudiantes de pregrado, y tienen como responsabilidad orientar el proceso escritural de los estudiantes que solicitan apoyo a la escritura. Calupso y Rincones (2010) afirman que para desarrollar el proceso de tutoría con éxito es necesario que el tutor genere un ambiente de confianza y seguridad, en el que prime el texto académico como creación y las metas del estudiante como escritor.

Calupso y Rincones (2010) realizaron una investigación titulada "Centro de Escritura Uniminuto: una alternativa para mejorar las competencias de lectura y escritura del estudiante, más allá de las aulas", durante 2009 y 2010, para optar al título de comunicadores sociales y periodistas. Dentro de 
sus conclusiones se plantean lo siguiente: el centro se ha proyectado como un espacio de asistencia voluntaria, para fortalecer las prácticas de lectura y escritura; las asesorías personalizadas han permitido a los estudiantes acercarse a su texto desde su forma y contenido; el acompañamiento del tutor ha sido importante para identificar las fortalezas y falencias del escrito, y para entender los elementos de una tipología específica.

Dentro de las búsquedas realizadas en la web, se encontró que desde el Centro de Escritura Uniminuto se han realizado intentos por incorporar recursos digitales para su funcionamiento: 1. el blog ( $h t t p: / /$ centrodeescriturauniminuto.blogspot.com.co/) solo contaba con tres entradas en el 2013, y tres entradas en el 2014; no hay registro de nuevas actividades, y los comentarios hacen referencia a los horarios de atención e invitación a utilizar los servicios del CE. 2. El sitio web (https://sites.google.com/site/centrodeescriturauniminuto/home) solo tiene una bienvenida para las actividades del 2013, por parte de la coordinadora del CE. 3. La red social en Facebook (https://www.facebook. com/centrodeescritura.uniminuto) fue creada en el 2013 y su última publicación fue en el 2014. Como se logró identificar, a la fecha estas estrategias no han tenido continuidad.

\section{Centro de Apoyo para la Lectura, la Oralidad y la Escritura Diga, del Colegio DE Estudios Superiores de Administración, Bogotá}

\section{http://www.cesa.edu.co/El-Cesa/DIGA.aspx}

El Colegio de Estudios Superiores de Administración, de Bogotá, creó en el 2010 el
Centro de Apoyo para la Lectura, la Oralidad y la Escritura, el cual fue nombrado como Diga. Este centro funciona como complemento de las prácticas académicas, y busca fortalecer las habilidades comunicativas, hacer conscientes a los estudiantes sobre la influencia del dominio de las formas del lenguaje en la comprensión del entorno y sus repercusiones en los otros, y ser un punto de referencia para los profesores que necesitan apoyo en la planeación, desarrollo y calificación de actividades que involucren habilidades lingüísticas.

Entre los servicios que ofrece el centro, se encuentran los siguientes: 1 . asesorías; buscan que el estudiante, con la orientación del docente, identifique las falencias en sus escritos y las corrija. Este servicio está dirigido a estudiantes de pregrado y posgrado. 2. Tutorías; están determinadas en temáticas específicas, por ejemplo, puntuación, normas APA, tildes. Las tutorías son realizadas por profesores y tienen acceso a ella los estudiantes de pregrado. 3. Lectores pares; está conformado por un grupo de estudiantes de diferentes semestres, que tienen la responsabilidad de realimentar al estudiante escritor sobre su producción. 4. Cursos asociados con la escritura; trabajo colaborativo entre el profesor del curso y un profesor de Diga. 5. Asesoría a los profesores alrededor de temáticas en producción oral o escrita dentro de las diferentes materias, y revisión de textos.

Murillo (2012) expresa que entre los resultados del centro Diga, en el primer semestre del 2011 se encontró lo siguiente: se ha creado conciencia en los estudiantes de procesos de reescritura, asistencia voluntaria por parte de los estudiantes al centro de apoyo, 
fortalecimiento de actividades académicas alrededor de ensayos, unificación de criterios dentro de la institución para la entrega de trabajos escritos, reducción significativa del plagio entre los estudiantes, revisión de los cursos de las áreas de comunicación.

Como parte de los cursos asociados, Murillo (2012) realizó un estudio de caso sobre el fortalecimiento de la escritura académica, con apoyo de Diga, en dos grupos de la materia de Historia y Geografía. Participaron 31 estudiantes, quienes debieron escribir tres ensayos durante el semestre. Los puntos evaluados por Diga fueron los siguientes: 1 . estructura (60\%): introducción, hipótesis, argumentación, conclusión; 2. referencias (20\%): manejo de citas, bibliografía; 3. gramática (20\%): ortografía, puntuación, vocabulario. Los resultados arrojaron que hubo una mejoría significativa en cada uno de los puntos evaluados entre la escritura del primer y el tercer texto; además, el profesor del curso reconoció positivamente el acompañamiento brindado por Diga en el proceso de producción de los textos de los estudiantes.

\section{Centro de Escritura, de la Universidad ICESI, Cali}

\author{
http://www.icesi.edu.co/crea/cursos_e_progra- \\ mas_de_escritura.php
}

En la Universidad ICESI, de Cali, existe el Centro de Recursos para el Aprendizaje (CREA), el cual contempla entre sus responsabilidades y programas, las siguientes acciones: diplomados, seminarios, talleres, acompañamiento y asesoría para la labor de los profesores de la Universidad, el Centro de Apoyo para el Aprendizaje de las
Matemáticas Básicas (CAMB) y el Centro de Escritura. Así, el CE de la Universidad ICESI hace parte de una estrategia más amplia, que tiene dentro de sus frentes de trabajo el fortalecimiento de las competencias docentes y las competencias lógico-matemáticas y escriturales de los estudiantes de pregrado.

El Centro de Escritura Universidad ICESI es un espacio pensado para el asesoramiento a los estudiantes en el proceso de construcción de textos académicos. El CE comenzó su funcionamiento en el 2013. Los estudiantes pueden asistir a tutorías personalizadas sobre sus escritos. Esta estrategia usa la escritura para desarrollar en los estudiantes sus capacidades de análisis, síntesis, solución de problemas y pensamiento crítico, habilidades contenidas en la propuesta pedagógica de esta universidad.

Los tutores son estudiantes de pregrado, quienes apoyan los procesos de revisión, realimentación y escritura de los textos de los estudiantes. Los tutores cuentan con un sitio web (https://sites.google.com/sitel centroescrituraicesi/home), en el que se gestionan los procesos de tutorías: asignación de tutorías, ficha de tutoría, encuesta del servicio, base de datos de los tutores y una guía de trabajo del tutor; en esta se brindan herramientas para el desarrollo de la tutoría. Además, la comunidad universitaria se puede mantener actualizada de lo que pasa y proyecta el Centro de Escritura ICESI, por medio de la red social Facebook (https://www.facebook.com/CEscrituraUniversidadIcesi\#); este es un espacio activo, en el que se pueden reconocer publicaciones que invitan a las actividades del CE, y se promueven actividades institucionales y de la ciudad. 


\section{Centro de Lectura y ESCRITURA EN ESPAÑOL (CELEE), DE LA UNIVERSIDAD del Rosario, Bogotá}

http://www.urosario.edu.co/Centro-de-Lecturay-Escritura-en-EspanolIniciol

El Centro de Lectura y Escritura en Español hace parte del Centro Multicultural y Multilingüe de la Universidad del Rosario, de Bogotá. Su objetivo está orientado al buen uso de la lengua española en las actividades comunicativas, dentro de la comunidad académica. El centro está dirigido, principalmente, a reforzar la redacción, puntuación, tipologías textuales, retórica, citación y lógica en la producción de textos escritos y orales. El Centro de Lectura y Escritura en Español contempla, como un aspecto misional, las relaciones entre el pensamiento y la lengua española, considerando que los procesos de producción académica contribuyen al pensamiento y la reflexión. Por otra parte, se proyecta que en los años siguientes los egresados de la Universidad del Rosario, de Bogotá, se identifiquen por un alto estándar de calidad en el uso del español.

El centro dispone de servicios de asesoría presenciales a los estudiantes y profesores. Este servicio es ofrecido por profesores de la Universidad. Para solicitar la asesoría, se debe diligenciar un formulario de aproximación diagnóstica, seleccionar un horario de atención, según la disponibilidad del centro, y presentarse a la asesoría con el avance del escrito. La asesoría dura una hora.

En la página web del centro, la comunidad académica puede encontrar: 1 . el nombre de los asesores, con una breve presentación.
2. Material de apoyo sobre comprensión de lectura, conectores, el párrafo y la frase, ensayos académicos, la tilde según la RAE, listado básico de conectores, uso de las letras b y v, uso de las letras c-s-z, uso de las mayúsculas. 3. Presentaciones sobre el adjetivo, el adverbio, el sustantivo, el verbo y la puntuación en español. 4. Talleres de gramática, uso del gerundio y escritura. Vale anotar que todos los recursos que se encuentran disponibles en la web están bajo el formato pdf. No hay actividades que permitan el uso de recursos interactivos.

\section{Centro de Escritura de la Universidad del Cauca, Popayán}

\section{http://www.unicauca.edu.co/centroescritural}

El Centro de Escritura hace parte del programa de Fonoaudiología, de la Universidad del Cauca. Es un espacio pensado para potenciar las habilidades de escritura académica de los estudiantes de pregrado y posgrado; sin embargo, hay un servicio de asesoría diseñado para los profesores y administrativos. Tiene como visión convertirse en un referente a escalas institucional, regional y nacional, por el impacto en la formación de las competencias comunicativas de los estudiantes, en coherencia con los principios de la Universidad del Cauca.

Los estudiantes pueden solicitar el servicio de tutoría a partir de dos situaciones: 1. escritura de un texto en particular que ha sido asignado en una asignatura; 2 . apoyo para cualificar el desempeño en escritura académica, sin centrarse en un texto en particular. En el centro se aclara que la tutoría no es solo para los estudiantes que 
presentan dificultades en el proceso de escribir; pueden asistir también los estudiantes que deseen fortalecer sus habilidades en la producción de los textos. Igualmente, hay un servicio de asesoría a profesores que busquen orientaciones para el trabajo de la lectura y la escritura dentro de sus clases, o apoyo en el proceso de producción de un texto académico.

El servicio de tutoría es prestado por estudiantes de séptimo y décimo semestres del pregrado de Fonoaudiología, quienes son seleccionados por sus habilidades escriturales y buenas relaciones sociales; sin embargo, para desempeñarse como tutores es necesario participar previamente de un proceso de formación. Los tutores pueden apoyar a los estudiantes en las siguientes actividades: planificar el texto, escribir los borradores necesarios y revisar cada versión hasta que se obtenga el texto definitivo. El propósito de la tutoría es que el estudiante tome conciencia sobre su proceso de escritura.

Chois Lenis y Guerrero (2015) adelantaron una investigación dentro del CE de la Universidad del Cauca, que tenía como objetivo comprender las prácticas que se desarrollan dentro de las tutorías. Para ello, analizaron el diálogo que se generó entre el tutor y el estudiante en cinco tutorías, teniendo en cuenta qué hace y dice el tutor, sus estrategias, las relaciones establecidas entre el tutor y el estudiante, la apropiación de las ideas por parte del estudiante. Dentro de las conclusiones se afirma que no es posible señalar que exista una única forma de desarrollar las tutorías, se hace necesaria la formación y reflexión permanente por parte de los tutores.
En la página web del CE, los estudiantes pueden encontrar material de apoyo en formato pdf, en temáticas como el ensayo, la reseña, normas APA, signos de puntuación. Además, hay una sección sobre enlaces de interés que los remiten a otras entidades interesadas en la escritura y lectura, $\mathrm{u}$ otros CE nacionales e internacionales. Esta página tiene una información suficiente para que los estudiantes y profesores accedan de forma presencial a los servicios que ofrece el CE; sin embargo, fuera de los documentos en pdf, carece de posibilidades de interacción para el fortalecimiento de la escritura académica con apoyo de recursos digitales.

Chois Lenis (2015) manifiesta que dentro de los principales logros obtenidos por el Centro de Escritura de la Universidad del Cauca, desde su fundación en el 2013, se encuentran: 1 . desarrollo de más de mil tutorías a estudiantes de pregrado; 2 . alrededor de setenta talleres grupales sobre escritura académica; 3. diseño de materiales de apoyo a la escritura; 4 . visibilización del CE a escala institucional; 5 . aceptación de los servicios del CE por parte de los integrantes de la comunidad académica.

\section{Centro de Recursos para el Éxito Estudiantil (CREE), dE LA UNIVERsidad del Norte, SEDE BARRANQUILLA}

http://www.uninorte.edu.co/web/centro-de -recursos-para-el-exito-estudiantil-cree

El Centro de Recursos para el Éxito Estudiantil (CREE), de la Universidad del Norte, sede Barranquilla, es un centro que ofrece diversos servicios a los estudiantes 
de pregrado. Nació en el 2010, como un área estratégica de la Vicerrectoría Académica, que lidera las acciones institucionales de permanencia. Para ello, agrupó los servicios de apoyo académico existentes y los reorganizó en cuatro áreas: recursos de acompañamiento académico, orientación académica, competencias comunicativas y retención y éxito académico (Zúñiga, 2014).

El Centro de Recursos ofrece acompañamiento en más de 300 asignaturas que brinda la Universidad, en los diferentes programas de pregrado; con ello, busca intervenir en la retención y éxito académico de los estudiantes. Sin embargo, para mantener la coherencia dentro del recorrido que se viene haciendo sobre los $\mathrm{CE}$, se analizó el componente asociado con las competencias comunicativas, llamadas 'Tutorías Exprésate'.

La 'Tutoría Exprésate' es una asesoría personalizada, que apoya al estudiante de pregrado en el análisis y/o elaboración de textos académicos, en la comprensión de lecturas que se asignan en las materias, en la argumentación oral y en los procesos de investigación. Sin embargo, el estudiante podrá asistir a talleres sobre temas específicos, liderados por expertos, sobre habilidades de escritura, comprensión de lectura, expresión oral y presentación de trabajos. Durante los talleres, los expertos podrán apoyar al estudiante en sus requerimientos académicos dentro de alguno de los temas enunciados. Vale anotar que las tutorías y talleres también se ofrecen para lenguas extranjeras, según la programación del Centro de Recursos.

\section{Centro de Lectura y EsCRITURA (CELEE), DE LA Universidad Autónoma de Occidente, Sede Cali}

http://www.uao.edu.co/comunicacion-social/ departamento-de-lenguaje/asesoria-departamento-de-lenguaje

El Centro de Lectura y Escritura (CELEE) hace parte del Departamento de Lenguaje, del programa de Comunicación Social, de la Universidad Autónoma de Occidente, sede Cali. Este es un espacio pensado para el acompañamiento de los procesos de escritura académica de los estudiantes de pregrado y posgrado, no necesariamente para las actividades asociadas con las asignaturas; la asesoría puede nacer de un interés del estudiante por cualificar sus procesos de producción de textos. La información del CE se encuentra en la página web institucional, y no hay recursos digitales para el trabajo de escritura.

Roldán Morales y Arenas Morales (2016) realizaron una investigación con el propósito de caracterizar las tutorías que se desarrollan en el CELEE. Para ello, realizaron un análisis de los registros de las tutorías desarrolladas durante los tres últimos meses del segundo semestre del año 2014 y todo el primer semestre del 2015. Dentro de las conclusiones del estudio se encuentra que hay una concepción de la tutoría tanto remedial como de desarrollo de estrategias; sin embargo, predomina una tutoría colaborativa. Es importante generar espacios para socializar la filosofía sobre el acompañamiento de la escritura del CE con los tutores, y crear diálogos entre tutores sobre las estrategias que aplican y los resultados que obtienen. 


\section{Centro de Escritura,} Lectura y Oralidad Académica (CELOA), de la UNIVERSIDAD SANTIAgO DE Cali, Cali

http://www.usc.edu.co/index.php/portafolio -de-servicio-por-componentes

El Centro de Escritura, Lectura y Oralidad Académica (CELOA) hace parte del programa de Fonoaudiología de la Universidad Santiago de Cali. Es un espacio para promover las competencias comunicativas orales y escritas en los estudiantes de pregrado con un perfil de vulnerabilidad de repitencia y/o deserción académica. El estudiante puede acceder a orientación para el mejoramiento de las competencias en lectura, escritura y oralidad académica. Además, el CE tiene diseñado un taller presencial sobre oralidad académica, que se ofrece semanalmente a la comunidad universitaria.

Trujillo, Torres, Montaño y Hurtado (2015) afirman que el CELOA es el resultado de una investigación que se diseñó en cinco pasos: 1. identificación de las necesidades e implementación del servicio; 2 . formalización del centro; 3. proyección; 4. movilización estudiantil; 5 . investigación, acción y evolución. Dentro de los logros del proceso, se encuentra que los estudiantes del programa de Fonoaudiología cuentan con herramientas pedagógicas para abordar la escritura, la lectura y la oralidad dentro de sus prácticas académicas.

\section{Centro de Producción Textual, de la Fundación UNIVERSITARIA UNITRÓPICO, YOPAL}

http://www.unitropico.edu.co/index.php/ centro-de-produccion-textual

El Centro de Producción Textual de Fundación Universitaria Unitrópico, de Yopal, es un centro especializado en la atención a estudiantes, personas naturales y jurídicas que requieran mejorar la producción y calidad de sus textos. Tiene por misión fortalecer la redacción de los estudiantes de pregrado y posgrado, mediante cursos del Programa de Atención y Seguimiento a Estudiantes (PASE); también, apoyar a docentes y administrativos en lo referido a la composición de textos de divulgación científica, académica e informativa.

Dentro de las actividades que orienta el CE se encuentra la promoción de la escritura, mediante talleres, seminarios y congresos de producción textual. Por otra parte, un aspecto distintivo del CE es su servicio de acompañamiento a entidades gubernamentales y personas naturales o jurídicas de la región de la Orinoquía colombiana, en el fortalecimiento de las estructuras textuales.

\section{Centro de Escritura Telar, de la Universidad Colegio Mayor de Cundinamarca, Bogotá}

http://www.unicolmayor.edu.co/nuevo/index. php?idcategoria $=3832$

El Centro de Escritura Telar, de la Universidad Colegio Mayor de Cundinamarca, de Bogotá, tiene como propósito fortalecer los 
procesos de escritura en la comunidad educativa. Cuenta con tres frentes de trabajo: personal administrativo, profesores y estudiantes. Los estudiantes pueden acceder a asesoría o talleres sobre lectura y escritura académica, ofrecido por profesores de la Universidad. Los profesores tienen acceso a asesorías y cursos o seminarios sobre divulgación científica. Cada semestre se publica en la página web de la universidad la programación del CE, y los integrantes de la comunidad educativa tienen la posibilidad de inscribirse para participar de las actividades.

\section{Centro Virtual de}

ESCRITURA, LENGUAJE $Y$ EXPRESIÓN (CVELE), DE LA UNIVERSIDAD NACIONAL

\section{Abierta y a Distancia, Colombia}

https://academia.unad.edu.co/centro-virtual-de-escritura-lenguaje-y-expresion?showa$l l=\mathcal{E}$ star $t=1$

El Centro Virtual de Escritura, Lenguaje y Expresión (CVELE) hace parte de la Facultad de Ciencias de la Educación, de la Universidad Nacional Abierta y a Distancia (UNAD). El centro tiene como misión apoyar a la comunidad académica de la UNAD para el fortalecimiento de las habilidades comunicativas en lectura, escritura, expresión artística, pruebas de Estado Saber Pro y competencias digitales. Además, busca ser reconocido como un ambiente de aprendizaje virtual especializado en el fortalecimiento de la lectura, la escritura y las actividades del área del lenguaje, a escalas nacional y latinoamericana.
El CVELE presenta los siguientes frentes de trabajo:

- Formación integral: En esta sección, los estudiantes pueden ingresar a una serie de cursos orientados a la lectura crítica, comunicación escrita, competencias ciudadanas, razonamiento cuantitativo, competencias básicas. Posee la estructura de las competencias genéricas de las pruebas Saber Pro. Los talleres y cursos están en la plataforma Blackboard.

- Investigación: Contiene un manual para presentar trabajos académicos en la UNAD. Este manual, en formato pdf, es un insumo para el desarrollo de procesos de investigación y redacción de textos de divulgación científica. Está orientado a la comunidad académica en general.

- Proyección social: Enfocada al desarrollo de las habilidades comunicativas de los profesores. Estos podrán participar en el curso de competencias comunicativas docentes, el cual se encuentra en la plataforma Blackboard.

- Internacionalización: Existe una serie de enlaces que remiten a instituciones interesadas en el tema de lectura y escritura. Se podrá encontrar el Centro de Escritura del Tecnológico de Monterrey, México; El Centro de Escritura de la Universidad de Puebla, México; La Asociación Internacional de Centros de Escritura; el Centro de Redacción Multidisciplinario de la Universidad Interamericana del Recinto Metro, Puerto Rico, entre otros. 
- Inclusión: Es un componente diferenciador de otros CE. En esta parte se puede encontrar una serie de videos traducidos a la lengua de señas, entre ellos: ¿cómo escribir un párrafo?, lectura comprensiva, el párrafo, comprensión oral y escrita, ¿cómo realizar un escrito? Esta parte responde a los compromisos de inclusión de la UNAD. Además, existe otra página web orientada a los estudiantes sordos (http://unadsorda.wixsite.com/cele).

- Innovación: En esta sección, la comunidad educativa puede encontrar una serie de objetos virtuales de aprendizaje (OVA), orientados a las competencias comunicativas y lógico-matemáticas. Entre los OVA se pueden encontrar recursos de argumentación, competencias comunicativas docentes, comprensión de lectura, lectura de imágenes, puntuación, ortografía, matemáticas cotidianas, pensamiento lógico matemático.

- Tutoría: Los estudiantes pueden acceder a un servicio de asesoría de trabajos académicos, de manera virtual. Para ello, deben estar registrados en la plataforma de la UNAD, solicitar el espacio y asistir con los avances en la producción del escrito. Hay que tener presente que, como lo afirman Pedraza Ramírez y Cabrera Otálora (2015), la población estudiantil de la UNAD complejiza más el proceso de acompañamiento del CVELE, debido al número de estudiantes, la dispersión geográfica, la diversidad cultural y de capacidades cognitivas, y las disciplinas de estudio.

El CVELE es un centro que utiliza las tecnologías digitales para el fortalecimiento de la lectura, escritura, lógica matemática y competencias básicas, en la comunidad educativa de la UNAD. El uso de estos recursos se apoya en la infraestructura tecnológica que posee la UNAD en el territorio colombiano. Vale anotar que los recursos para la formación integral, proyección social y tutoría son cerrados; es decir, solo tienen acceso los estudiantes y profesores de la UNAD. Los demás recursos se encuentran disponibles para la comunidad en general.

\section{Centro de Español, de la FUNDACIÓN UNIVERSITARIA Konrad Lorenz, Bogotá}

\section{http://www.konradlorenz.edu.co/es/estudiantes/ instituto-de-humanidades/centro-de-espanol. html}

El Centro de Español hace parte del Instituto de Humanidades, de la Fundación Universitaria Konrad Lorenz, sede Bogotá. Fue creado en el 2011, con el fin de fortalecer los alcances de la asignatura de Habilidades Comunicativas. Sin embargo, sus intereses se han ampliado en potenciar las habilidades comunicativas en las diferentes asignaturas que se ofrecen en la Universidad. El centro está coordinado y orientado por profesores que se encuentran adscritos al Instituto de Humanidades, y que se desempeñan en áreas del lenguaje.

El Centro de Español ofrece los servicios de tutoría y talleres a los estudiantes de pregrado. Las tutorías de lectoescritura ofrecidas están orientadas para despejar inquietudes y potenciar estas habilidades. Cada semestre se programan y divulgan los talleres que se van a ofrecer; los estudiantes se deben inscribir previamente para participar. Se 
encuentran talleres sobre normas APA, signos de puntuación, lectura comprensible, búsquedas de información en internet, entre otros. Además, cuenta con una página de recursos llamada Konpalabra, para la escritura académica; allí se aloja una serie de guías sobre la lectura y la escritura, para que la comunidad en general pueda consultar.

\section{Centro de Estudios en LECTURA Y ESCRITURA (CELEE), DE LA UNIVERSIDAD EAFIT, Medellín}

\section{http://www.eafit.edu.co/celee}

El Centro de Estudios en Lectura y Escritura de la Universidad EAFIT está adscrito al Departamento de Humanidades. Tiene como propósito el fortalecimiento de las competencias de lectura y escritura de los estudiantes de pregrado y posgrado, de profesores y empleados de la Universidad; sin embargo, a sus servicios pueden acceder tanto la comunidad académica como empresarial de la región. Dentro de los servicios se encuentran cursos, talleres, asesorías y consultorías, con énfasis en la lectura y la escritura. Está coordinado y orientado por profesores especializados en el área del lenguaje, pertenecientes al Departamento de Humanidades de la Universidad EAFIT.

El CELEE ofrece los siguientes servicios:

- Laboratorio de lectura y escritura: Espacio de intercambio académico para resolver dudas, e identificar y buscar soluciones a los problemas de lectura y/o escritura. Este laboratorio está dirigido a estudiantes de pregrado y posgrado, profesores y empleados de la Universidad EAFIT. Es un espacio de tutoría de textos académicos. Para acceder al servicio, el interesado debe diligenciar un formulario, para que se le pueda agendar y confirmar la cita. La tutoría es dirigida por un profesor del área del lenguaje, y se desarrolla de manera presencial.

- Talleres de entrenamiento de Pruebas Saber Pro: Curso en fortalecimiento en competencias en lectura y escritura. Dirigido a estudiantes de pregrado, entre octavo y décimo semestres, que deban presentar la prueba Saber Pro.

\section{- Cursos Núcleo de Formación Institu-} cional: Lo conforman tres cursos: Análisis Textual, Prácticas Textuales y Edición Textual, cuyos objetos de estudio son los textos hablados y escritos.

- Cursos de escritura académica para posgrado: Curso-taller, cuyo objeto de estudio son los textos académicos. Se centra en el proceso de construcción y composición de dichos textos (monografía, artículo científico y tesis de investigación). Ejemplo de curso: posicionamiento del autor a través de los verbos.

- Cursos para empleados y docentes: Enfocados al fortalecimiento y uso efectivo de la lectura y la escritura en las actividades diarias. Dirigido a profesores y empleados de la Universidad. Asesoría y acompañamiento a los docentes para la planeación, el desarrollo y la calificación de las actividades que involucren lectura y escritura en sus materias 
específicas. Ejemplo: curso de gramática y legibilidad.

- Prueba de clasificación: Al ingresar en la Universidad EAFIT, los estudiantes presentan una prueba de habilidades comunicativas, con el objetivo de determinar el grado de competencias de lectura y escritura, y ubicarlos, de acuerdo con sus competencias, en uno de los tres cursos de habilidades comunicativas. Esto, con el fin de reforzar en los estudiantes de pregrado aquellos aspectos que no dominen.

- Servicios a la comunidad: Asesoría y consultoría a instituciones educativas y empresas de la región interesadas en el diseño de cursos y talleres de habilidades comunicativas, pruebas de ingreso, autodiagnóstico o clasificación de sus estudiantes/empleados; seminarios en habilidades comunicativas, y elaboración de material didáctico para la enseñanza de la lectura y la escritura.

En la página web del CELEE, la comunidad en general puede encontrar un enlace sobre buenas prácticas en lectoescritura, que tiene tres componentes: 1. preguntas frecuentes; serie de guías en pdf sobre algunos aspectos relevantes de la lectura y la escritura. 2. Repositorio de las presentaciones utilizadas en los diferentes talleres ofrecidos por el CELEE. 3. Claves para la lectura y escritura; se trata de una serie de videos en los que se presentan algunas estrategias para el desempeño y desarrollo de los procesos lectores y escriturales.
Programa de Lectura y

Escritura Académicas, de

LA UNIVERSIDAd NACIONAL DE

Colombia (Programa lea

EN LA UN), SEDES Bogotá,

Medellín, Manizales,

Palmira, Caribe, Orinoquía, Amazonía y Tumaco

http://lea.unal.edu.co/

El Programa de Lectura y Escritura Académica, de la Universidad Nacional de Colombia (Programa LEA en la UN), inició sus actividades en el 2013. Uno de sus propósitos es apoyar a los estudiantes de pregrado de la Universidad en el fortalecimiento de las competencias comunicativas de lectura y escritura. En el programa, los procesos de lectura y escritura académicas se entienden como una práctica que atraviesa el currículo y se da desde todas las disciplinas. Los principios del programa se fundamentan en el trabajo colaborativo, aprendizaje autónomo y reconocimiento de la diversidad lingüística. Santos (2014) afirma que, a partir de la experiencia de investigación acción participativa, sobre la que se consolidó el Programa LEA en la UN, se extrapola una comprensión profunda del proceso, base para la transformación de las prácticas de lectura y escritura de los integrantes de la comunidad universitaria.

Dentro de los objetivos específicos del programa se encuentran sensibilizar a los profesores de la Universidad frente a su responsabilidad en los procesos de lectura y escritura en las asignaturas, mejorar las competencias de lectura y escritura de estudiantes pertenecientes a comunidades vulnerables, y conformar una comunidad 
virtual de aprendizaje en torno a la lectura y escritura académicas. El programa se fundamenta teóricamente en tres líneas de estudio de la escritura para la educación superior: la alfabetización académica, la literacidad crítica y la geopolítica de la escritura académica.

El grupo de trabajo del programa está conformado por: 1. una coordinadora, responsable del funcionamiento administrativo y pedagógico del programa. 2. Un grupo de apoyo, con profesionales en el área del lenguaje y la comunicación, responsables de asesorar a los profesores de la Universidad y orientar a los estudiantes auxiliares. 3. Auxiliares; se trata de estudiantes de últimos semestres de varias carreras, con habilidades para la lectura y escritura, quienes son responsables del proceso de tutoría académica de los estudiantes.

La población objeto del programa son los estudiantes y profesores. Los estudiantes pueden asistir a tutorías académicas; para ello, deben solicitar una cita por medio del correo electrónico, en el que expliquen los motivos para requerir la tutoría; esperar la confirmación de la tutoría, presentarse en el día y la hora indicados, con el avance en la producción escritural. Vale anotar que actualmente se está diseñando un espacio en la plataforma Moodle para la atención de tutorías virtuales. Por otra parte, los profesores pueden solicitar acompañamiento de uno de los profesionales de apoyo para el trabajo de la lectura y escritura dentro de sus asignaturas, al tiempo que asisten a un seminario-taller sobre esta temática.

En la página web del programa, la comunidad universitaria encontrará objetos virtuales de aprendizaje (OVA) sobre la presentación y producción de un tipo de texto académico que hace parte del currículo de la Universidad; por ejemplo, el ensayo, la reseña descriptiva, la reseña crítica. Además, encontrará otros recursos pensados para aprender sobre escritura académica y tipología de textos académicos, mejorar la redacción y la ortografía, mejorar las estrategias de lectura y referenciación; se trata de enlaces que remiten a otras páginas web de recursos digitales sobre la temática, $u$ otros CE.

\section{Centro de Escritura, de la UNIVERSIDAd EAN, Bogotá}

http://noticias.universidadean.edu.co/seccion/ nuevo-centro-de-escritura-en-la-universidad-ean.html

El Centro de Escritura, de la Universidad EAN, de Bogotá, hace parte de la Facultad de Humanidades y Ciencias Sociales. Comenzó sus servicios en el 2016; entre ellos se cuentan tutorías para la escritura, lectura y métodos de estudio, para los estudiantes de pregrado de cualquiera de los programas que ofrece la Universidad. Dentro de las tutorías se pueden abordar dificultades de redacción, ortografía, organización de ideas y planeación de los trabajos escritos. El estudiante que requiera una tutoría podrá escribir un correo electrónico solicitando el servicio; para ello deberá indicar el tema que desea trabajar durante la tutoría. Posteriormente, recibirá una respuesta de los tutores para confirmar la fecha y la hora del encuentro. 


\section{Centro de Escritura Juanambú, del Colegio Juanambú, Cali}

https://sites.google.com/a/juanambu.edu.co/ centro-de-escritura-juanambu/home

El Centro de Escritura del Colegio Juanambú, de Cali, es un espacio virtual, diseñado para los estudiantes, docentes y padres de familia, orientado a potenciar la escritura académica y creativa de los niños y jóvenes. Es el único $C E$ que se ha identificado orientado a estudiantes de educación básica y media. Sin embargo, desde el 2014 no se registra alguna actividad nueva, y los materiales que se publican son tomados de CE que fueron pensados para la educación superior.

El CE está apoyado en una página web que contiene los siguientes recursos: 1 . noticias; serie de enlaces que remiten a publicaciones en prensa sobre la lectura y la escritura en la escuela. 2. Tutorías, las cuales se definen como un acompañamiento para mejorar la comprensión y producción de textos. Aunque existe un calendario, no hay registro de ninguna tutoría desarrollada; además, el enlace para la solicitud de la tutoría se encuentra inactivo. 3. Material de apoyo; contiene enlaces que remiten a otras entidades que tienen dentro de sus intereses los procesos de lectura y escritura en la escuela, igualmente se encuentran enlaces que remiten a otros CE. 4. Recursos para estudiantes, guías, recomendaciones, ejercicios y ejemplos sobre diferentes temáticas relacionadas con el proceso de escritura académica, en formato pdf. Son insumos que se tomaron de otros CE. No hay una construcción propia. 5. Recursos para la familia; remite a una página web que realiza actividades de promoción de lectura y escritura en familia. 6. Recursos para docentes, material en formato pdf para el trabajo de la lectura y la escritura dentro de las áreas del lenguaje. Es un insumo tomado de otros CE; no hay construcción propia.

\section{Perspectiva de los CE en Colombia}

Como se puede observar en la figura 1, el 47,4 $\%$ de los CE que se identificaron se encuentran ubicados en Bogotá, ciudad que abarca el mayor número de estos centros en Colombia; seguido de Cali, con el 26,3 \%, y Medellín, con el 10,5\%. Vale anotar que el Centro Virtual de Escritura, Lenguaje y Expresión (CVELE) de la Universidad Nacional Abierta y a Distancia tiene impacto en todas sus 53 sedes. Todo esto permite reconocer que, a pesar del poco tiempo que llevan los CE en Colombia, la incorporación de esta estrategia en la educación superior viene en expansión.

En las instituciones educativas se ha determinado una estructura administrativa para el funcionamiento de los CE. En el contexto colombiano, se identificó que el 63,2 \% de los CE dependen directamente de facultades o departamentos del área del lenguaje. El $10,5 \%$ están coordinados por facultades o departamentos diferentes al área del lenguaje; por ejemplo, el Centro Virtual de Escritura, Lenguaje y Expresión (CVELE), de la Universidad Nacional Abierta y a Distancia, que está asociado con la Facultad de Educación. El 15,8 \% de los CE dependen de otros centros de recursos; por ejemplo, el Centro de Lectura y Escritura en Español de la Universidad del Rosario hace parte del Centro Multicultural y Multilingüe. El 10,5 \% de los CE dependen de otras unidades administrativas; 
por ejemplo, el Centro de Español de la Universidad de los Andes hace parte de la Vicerrectoría de Asuntos Académicos. Se observa que continúan prevaleciendo los departamentos del lenguaje como responsables de los procesos de lectura y escritura dentro de las instituciones; sin embargo, los CE promueven la mirada transversal y disciplinar de las habilidades comunicativas (figura 2).

Figura 1. Ubicación geográfica de los CE en Colombia

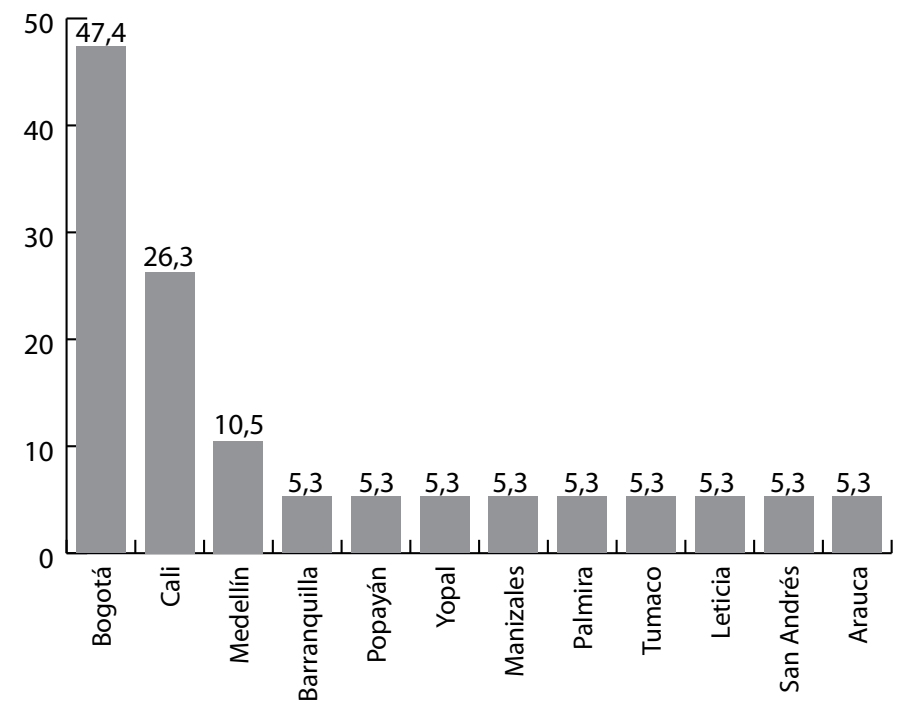

Fuente: Elaboración propia.

Figura 2. Dependencia administrativa de los CE en Colombia

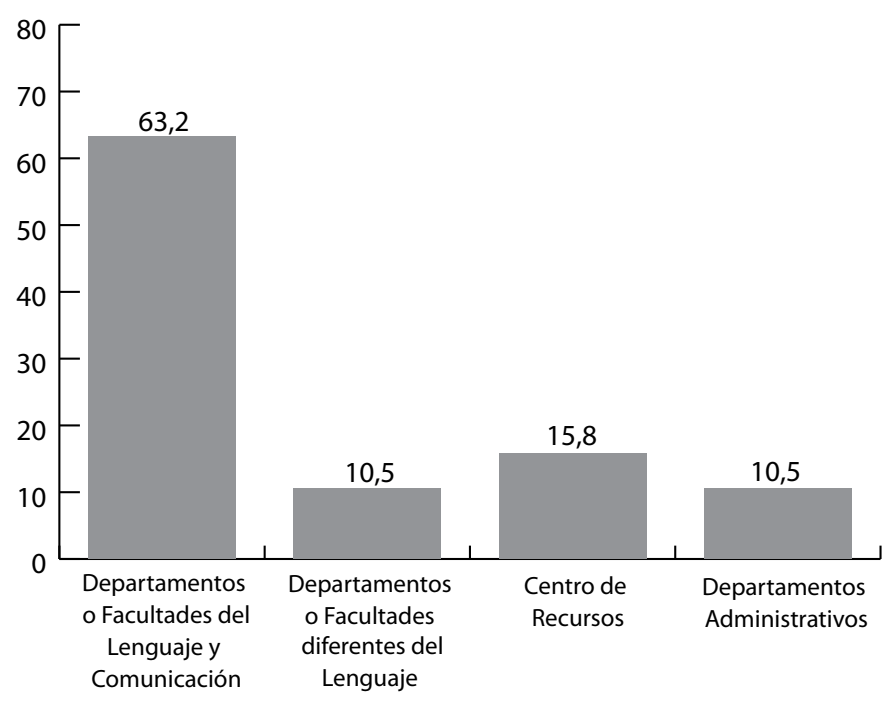

Fuente: Elaboración propia. 
En el 89,5\% de los CE se considera la escritura como un proceso complejo que requiere acompañamiento y apoyo. Sin embargo, en el 10,5\% de ellos prevalece un énfasis en lo lingüístico y gramatical, con un interés en brindar instrucciones a los estudiantes sobre cómo utilizar los signos de puntuación, la correcta escritura de las palabras y las condiciones mínimas al momento de escribir en un género discursivo. Lo que requeriría una revisión sobre la mirada de la escritura para una sociedad como la colombiana.

Respecto a los propósitos misionales de los $\mathrm{CE}$, en las instituciones educativas se pudo identificar que en el $100 \%$ de los casos, la motivación que dio origen a estos espacios fue el fortalecimiento de la escritura académica. Pero a este interés se le han agregado otras tareas misionales, así: el 73,7 \% de los CE consideran importante trabajar paralelamente la lectura y la escritura; por ejemplo, el Programa de Lectura y Escritura Académicas de la Universidad Nacional de Colombia (Programa LEA en la UN) considera imprescindible apoyar estas dos habilidades para el fortalecimiento de las competencias académicas. El 15,8 \% de los CE tienen dentro de sus propósitos misionales aportar a la expresión oral; en ese caso, el Centro de Apoyo para la Lectura, la Oralidad y la Escritura Diga, del Colegio de Estudios Superiores de Administración, de Bogotá, incorporó dentro de sus responsabilidades potenciar la expresión oral dentro de las prácticas académicas de las asignaturas que ofrece la universidad (figura 3).

El 26,3 \% de los CE consideran necesario trabajar los procesos de pensamiento; así, el Centro de Escritura de la Universidad
ICESI, de Cali, aporta al modelo pedagógico institucional, en cuanto busca estrategias para que los estudiantes que asistan a los servicios del centro puedan fortalecer sus habilidades de pensamiento crítico. El 36,8 \% de los CE tienen dentro de sus propósitos procurar que los estudiantes permanezcan dentro de sus programas académicos, debido a que consideran que una de las causas de deserción son las dificultades de lectura y escritura que se presentan dentro de las prácticas académicas. El 15,8 $\%$ de los CE tienen intereses que favorecen los procesos de inclusión escolar; para ello, buscan atender poblaciones que pertenecen a poblaciones vulnerables, indígenas o excluidos de la sociedad. El 15,8 \% de los CE han acompañado los procesos de lectura y escritura, y han dado orientaciones en métodos de estudio; por ejemplo, el Centro de Escritura de la Universidad EAN, de Bogotá, acompaña los procesos de tutorías y orientaciones en métodos de estudios para mejorar las estrategias de autoaprendizaje.

En el $100 \%$ de los CE, la tutoría académica es considerada como un proceso de acompañamiento y apoyo al proceso de producción de textos; esto concuerda con el panorama mundial planteado por Calle Álvarez (2016). Sin embargo, hay diferencias en el manejo de las tutorías. Así, en el 52,6\% de los centros, los tutores son estudiantes de pregrado; en el $26,3 \%$, estudiantes de posgrado, y en el 57,9\%, profesores del área del lenguaje. Esto permite visibilizar que la mirada sobre los pares académicos viene tomando fuerza en Colombia, pero las orientaciones y observaciones sobre los procesos de escritura de los profesores siguen teniendo un alto impacto sobre los estudiantes. 
Figura 3. Principios misionales de los CE en Colombia

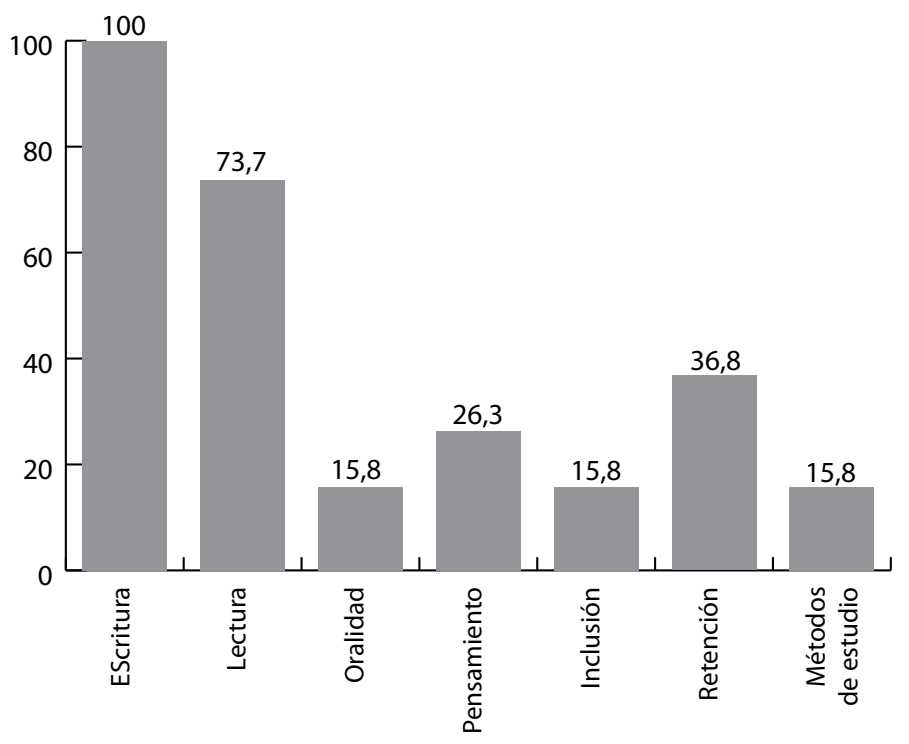

Fuente: Elaboración propia.

En la misma dirección, del 38,5 \% de los CE donde los estudiantes de pregrado pueden ser tutores, el $60 \%$ les exige una serie de actividades formativas, que en algunos casos puede durar un semestre académico, como en el Centro de Español de la Universidad de los Andes o el Centro de Escritura Javeriano. Además, esta formación va acompañada de un apoyo por parte de los coordinadores de los $\mathrm{CE}$, o de profesionales del área del lenguaje. Esto permite evidenciar que el proceso de tutoría requiere personas idóneas para desempeñar las funciones, al tiempo que precisa de un acompañamiento permanente por parte de los responsables del CE.

Frente a la población beneficiaria de los servicios de los CE, se ha encontrado que el 94,7\% de los usuarios son los estudiantes de pregrado; el 57,9 \% son estudiantes de posgrado; el 73,7 \% son los profesores de cualquier grado educativo. Sin embargo, se destaca que algunos CE han incorporado servicios para otras poblaciones pertenecientes a la comunidad educativa; por ejemplo, el 26,3\% de los CE apoyan los procesos de lectura y escritura del personal administrativo de las universidades. El 5,3 \% tienen como objeto el apoyo a personas naturales externas a la institución; por ejemplo, el Centro de Producción Textual de la Fundación Universitaria Unitrópico, de Yopal, considera dentro de sus políticas apoyar a las personas naturales que requieran fortalecer sus habilidades escriturales académicas. El 10,5 \% considera importante el apoyo a empresas $u$ otras instituciones educativas; por ejemplo, el Centro de Estudios en Lectura y Escritura (CELEE), de la Universidad EAFIT, de Medellín, tiene dentro de sus objetivos apoyar y acompañar la creación de políticas institucionales o empresariales para el trabajo de las habilidades comunicativas, también están 
dispuestos a acompañar el diseño de recursos y pruebas sobre lectura y escritura. El 5,3\% de los CE tienen como población objeto los estudiantes de la educación básica y media; esto se debe a que al momento solo se ha reconocido el Centro de Escritura del Colegio Juanambú, de Cali. Vale anotar que este CE tiene dentro sus beneficiarios a los profesores y padres de familia de la comunidad educativa de Juanambú. El alcance de los CE para la atención de los usuarios está determinada por los propósitos misionales, los recursos humanos y financieros con que cuente el CE, además, del liderazgo y apoyo administrativo para la ejecución de las funciones asignadas (figura 4).

Los servicios ofrecidos por los CE se han diversificado; así, se ha identificado que el $100 \%$ de los CE han fundamentado sus servicios en la tutoría académica para los estudiantes de básica, media, pregrado y/o posgrado, dependiendo de la población objeto del CE. El 84,2 \% de los CE acompañan los procesos de tutoría académica de talleres; por ejemplo, el Centro de Escritura, Lectura y Oralidad Académica (CELOA), de la Universidad Santiago de Cali, tiene diseñado un taller de oralidad académica que se ofrece cada semana a los estudiantes de la Universidad. El 73,7 \% de los CE tienen servicio de asesoría para los profesores en el fortalecimiento de sus habilidades escriturales y en el acompañamiento didáctico para el trabajo de lectura y escritura dentro de las asignaturas. Igualmente, el 63,2 \% de los CE tienen talleres diseñados para los profesores y/o personal administrativo de la Universidad; así, el Centro de Escritura Telar, de la Universidad Colegio Mayor de Cundinamarca, de Bogotá, ofrece a los profesores un seminario-taller de divulgación científica.

Figura 4. Población beneficiaria de los CE en Colombia

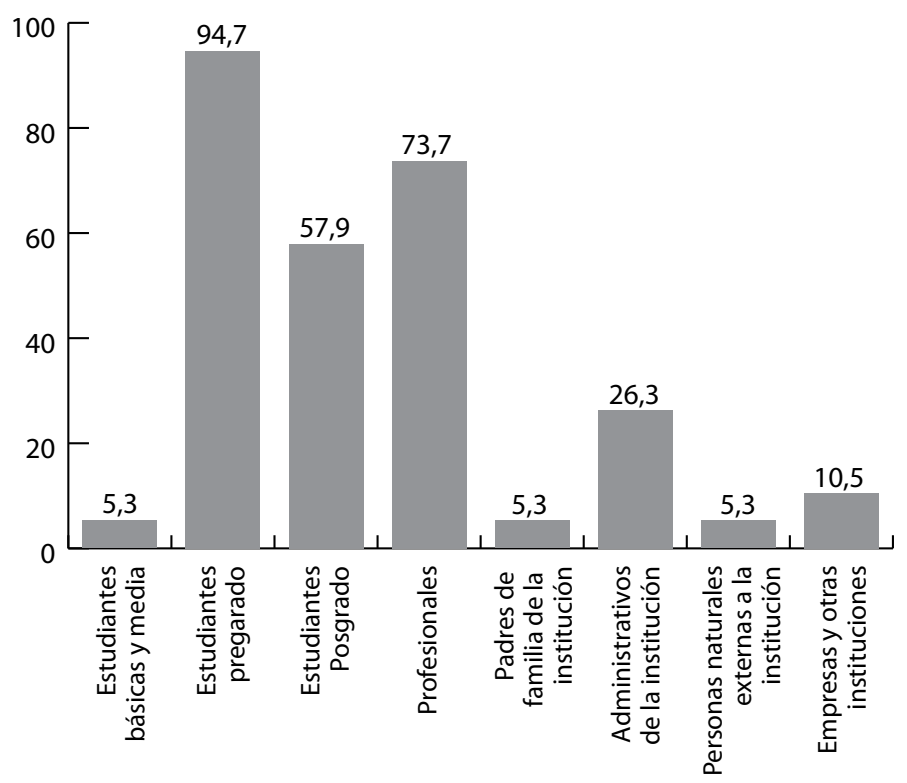

Fuente: Elaboración propia. 
El 63,2 \% de los CE tienen un espacio de recursos digitales en sus páginas web; estos pueden ser guías en pdf, videoconferencias, enlaces a otras entidades interesadas en los procesos de lectura y escritura, o a otros $\mathrm{CE}$, nacionales o internacionales. El Centro Virtual de Escritura, Lenguaje y Expresión (CVELE), de la Universidad Nacional Abierta y a Distancia, de Colombia, tiene recursos digitales para el acompañamiento de la lectura y escritura académica de estudiantes sordos, por ejemplo. El 5,3\% de los CE cuentan con acompañamiento a entidades externas para el diseño de pruebas sobre lectura y escritura. Los servicios de los CE siguen reconociendo la importancia de la tutoría para los estudiantes; además, han valorado y acompañado las prácticas de aula de los profesores (figura 5).

Los centros de escritura tienen un espacio en la web de las instituciones o han creado sus propias páginas. El 84,2 \% de los CE requieren potenciar los recursos digitales para el apoyo de la lectura y escritura, y superar los formatos pdf o su uso para agendar citas de tutoría, con el fin de aprovechar las posibilidades multimodales digitales de los espacios web. Se destaca el Centro Virtual de Escritura, Lenguaje y Expresión (CVELE), de la Universidad Nacional Abierta y a Distancia, de Colombia, pues ha aprovechado los recursos que le ofrece la plataforma Blackboard para diseñar talleres, y tiene, además, un servicio de tutoría virtual. Igualmente, el Programa de Lectura y Escritura Académicas de la Universidad Nacional de Colombia (Programa LEA en la UN) ha comenzado a diseñar un espacio virtual para el servicio de tutorías académicas. Los CE en el contexto colombiano se encuentran en un estado de inicio de incorporación de recursos digitales para el desarrollo de sus servicios.

Figura 5. Servicios de los CE en Colombia

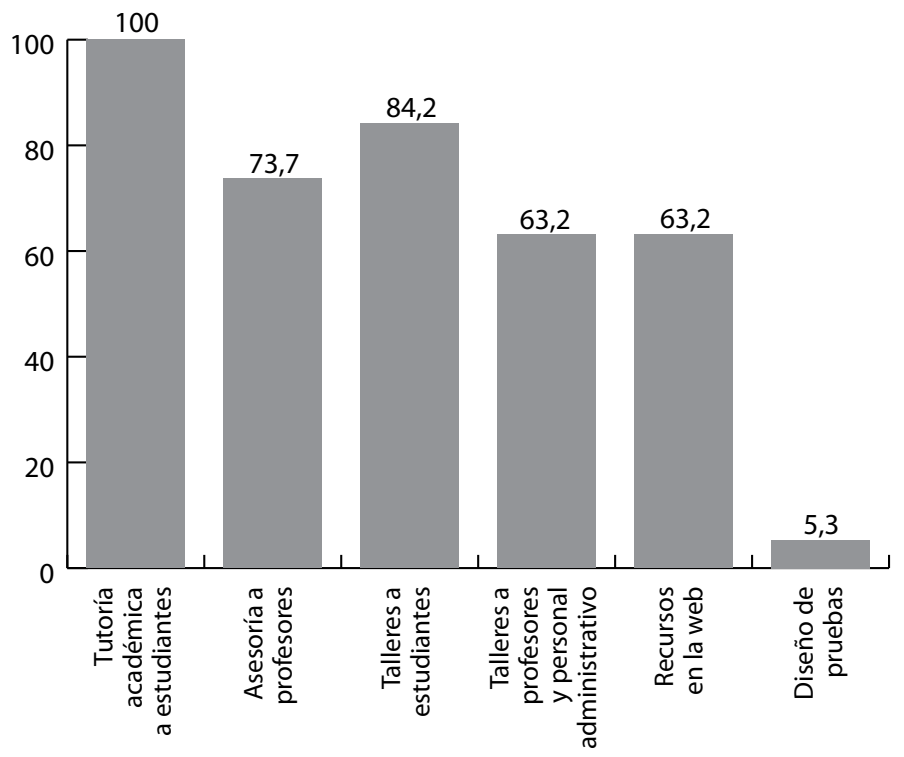

Fuente: Elaboración propia. 
Se describieron 18 centros de escritura en diferentes universidades y 1 de una institución educativa de básica y media, del escenario colombiano. Los centros de escritura en Colombia son una estrategia reciente, que se ha venido pensando para cumplir con el propósito de fortalecer los procesos de escritura académica en los estudiantes, especialmente de pregrado. Algunos de ellos amplían sus posibilidades, al incorporar habilidades como la lectura y el pensamiento crítico. Sin embargo, una de las metas sigue estando enfocada en el acercamiento de la escritura como una posibilidad para adquirir y acceder a competencias que requiere un profesional en el contexto social, económico y político de una nación.

\section{REFERENCIAS}

Alfonzo, I. (1994). Técnicas de investigación bibliográfica. Caracas: Contexto Ediciones.

Calle Álvarez, G. Y. (2016). Cartografía de los centros de escritura: un estado del arte. [Con] textos, 5(17), 29-39.

Calupso Cano, S. T., y Rincón Machena, M. A. (2010). Centro de escritura Uniminuto una alternativa para mejorar las competencias de lectura y escritura del estudiante fuera de las aulas. Trabajo de pregrado. Recuperado de http://repository.uniminuto.edu:8080/ xmlui/bitstream/handle/10656/1153/ TC_CalupsoCanoShionek_2010.pdf? sequence $=1 \&$ is Allowed $=y$

Chois Lenis, P. M. (2015). Centro de Escritura Universidad del Cauca CEUC. En: Molina Natera, V. (Editora). Panorama de los centros y programas de escritura en Latinoamérica. Pontificia Universidad Javeriana, Cali, (p. 39-43). Recuperado de http://vitela.javerianacali.edu.co/
bitstream/handle/11522/8091/Libro_Panorama_Centros_Esc_completo.pdf?sequence $=1 \&$ is Allowed $=\mathrm{y}$

Chois Lenis, P. M., \& Guerrero, H. I. (2015). Los aportes de un tutor par de escritura académica. En: Molina Natera, V. (Editora). Panorama de los centros y programas de escritura en Latinoamérica. Pontificia Universidad Javeriana, Cali, (p. 153-163). Recuperado de http://vitela.javerianacali.edu.co/bitstream/handle/11522/8091/ Libro_Panorama_Centros_Esc_completo.pdf? sequence $=1$ \&is Allowed=y

Escallón Largacha, E. (2015). El Centro de Español de la Universidad de los Andes: una experiencia de aprendizaje auténtico de escritura en la educación superior. En: Escallón Largacha, E., y Forero Gómez, A. (autores-compiladores). Aprender a escribir en la universidad (p. XI-XIII). Bogotá: Universidad de los Andes.

Forero Gómez, A. (2015). Introducción al curso de Español. En: Escallón Largacha, E., y Forero Gómez, A. (autores-compiladores). Aprender a escribir en la universidad ( $p$. 73-76). Bogotá: Universidad de los Andes.

González, B. Y. (2015). Centro de Escritura Javeriana PUJ Bogotá. En: Molina Natera, V. (Editora). Panorama de los centros y programas de escritura en Latinoamérica (p. 63-70). Cali: Pontificia Universidad Javeriana. Recuperado de http://vitela.javerianacali. edu.co/bitstream/handle/11522/8091/Libro_Panorama_Centros_Esc_completo. pdf?sequence $=1$ \&isAllowed $=y$

González, B., y Vega, V. (2013). Prácticas de lectura y escritura en cinco asignaturas de diferentes programas de la Universidad Sergio Arboleda. Civilizar Ciencias Sociales y Humanas, 10(18), 101-116.

Lara Negrette, L. M. (2015) Acciones pedagógicas y secuencias de aprendizaje en las 
tutorías. En: Escallón Largacha, E., y Forero Gómez, A. (autores-compiladores). Aprender a escribir en la universidad (p. 159173). Bogotá: Universidad de los Andes.

Molina Natera, V. (2015). Estudio de caso: Centro de Escritura Javeriano PUJ Cali. En: Molina Natera, V. (Editora). Panorama de los centros y programas de escritura en Latinoamérica (p. 276-311). Cali: Pontificia Universidad Javeriana.

Montealgre, C., \& Morales, G. (2015). Introducción a los cursos E. En: Escallón Largacha, E., y Forero Gómez, A. (autores-compiladores). Aprender a escribir en la universidad (p. 3-6). Bogotá: Universidad de los Andes.

Moreno, E.,\& Baracaldo, N. (2015). La lectura y la escritura en la clase de Teoría del Aseguramiento: una experiencia del Centro de Escritura Javeriana Bogotá. En: Molina Natera, V. (Editora). Panorama de los centros y programas de escritura en Latinoamérica (p. 187-198). Cali: Pontificia Universidad Javeriana.

Murillo, J. (2012). La universidad y los procesos de lecto-escritura: centro de apoyo para la lectura, la oralidad y la escritura DIGA. Panorama, 6(10), 87-97.

Núñez, J. (2013). Una aproximación a los centros de escritura en Iberoamérica. Legenda, 17(17), 63-102.

Pedraza Ramírez, C. E., \& Cabrera Otálora M. I. (2015). Tutorías virtuales de escritura como espacio necesario en e-learning: propuesta para la UNAD sede Palmira. En: Molina Natera, V. (Editora). Panorama de los centros y programas de escritura en Latinoamérica (p. 223-234). Cali: Pontificia Universidad Javeriana.

Roldán Morales, C. A., \& Arenas Hernández, K. A. (2016). Características de las tutorías del Centro de Lectura y
Escritura de la Universidad Autónoma de Occidente: ¿Qué muestran los registros de atención? Grafía, 13(1), 100-114.

Santos, D (2014) Lectura y escritura académicas en la universidad: endogénesis e investigación acción participativa para el desarrollo curricular (Resumen, p. 24). En: Carlino, P., Cartolari, M., Molina, E., y Rosli, N. Libro de Resúmenes del Simposio Internacional Investigar la enseñanza de la lectura y escritura para aprender en distintas disciplinas del nivel medio y superior. In Simposio Internacional Investigar la enseñanza de la lectura y escritura para aprender. Grupo para la Inclusión y Calidad Educativas a través de Ocuparnos de la Lectura y la Escritura en todas las Materias, Biblioteca Nacional, Ciudad de Buenos Aires. Recuperado de http:// www.aacademica.org/simposio.internacional.investigar.la.ensenanza.de.la.lectura.y.escritura.para.aprender/2.pdf

Toloza, H., Barletta, N., \& Moreno, F. (2013). Una experiencia de acompañamiento en el proceso de enseñanza y aprendizaje de la lectura y la escritura en educación media. Zona Próxima, (19), 39-55.

Trujillo, A. S., Torres, M., Montaño, J. O., \& Hurtado, D. I. (2015). Una experiencia que fundamenta la responsabilidad de la fonoaudiología educativa en la Universidad. Revista Areté, 15(2), 6-16.

Zúñiga, M. (2014) Centro de recursos para el éxito estudiantil, una alternativa para el éxito de la generación Uninorte. IV Conferencia Latinoamericana sobre el abandono en la educación superior, Universidad de Antioquia, Medellín, Colombia. Recuperado de http://www.alfaguia.org/ www-alfa/images/PonenciasClabes/5/ ponencia_145.pdf 